Check for updates

Cite this: RSC Adv., 2017, 7, 56629

Received 21st October 2017

Accepted 9th December 2017

DOI: 10.1039/c7ra11632b

rsc.li/rsc-advances

\title{
Antifouling sulfonated polyamide nanofiltration hollow fiber membrane prepared with mixed diamine monomers of BDSA and PIP
}

\author{
Ben-Qing Huang, ${ }^{a}$ Zhen-Liang $\mathrm{Xu}, \mathbb{D}^{\mathrm{a}}{ }^{\mathrm{Hao}}$ Ding, ${ }^{a}$ Ming-Che Miao ${ }^{\mathrm{b}}$ \\ and Yong-Jian Tang (D) *a
}

A novel high-flux sulfonated polyamide nanofiltration (NF) hollow fiber membrane was made from the mixed monomers of 2,2'-benzidinedisulfonic acid (BDSA) and piperazine (PIP). By optimizing the preparation parameters (TMC concentration, reaction time and BDSA/PIP mixing ratio), the optimized NF membrane possessed a pure water permeability (PWP) of $49.1 \mathrm{~L} \mathrm{~m}^{-2} \mathrm{~h}^{-1}$ and a $\mathrm{Na}_{2} \mathrm{SO}_{4}$ rejection of 95.5\% under 4 bar. The molecular weight cut off (MWCO) of the BDSA/PIP NF membrane was about $400 \mathrm{Da}$. Furthermore, in a long-term antifouling test with $0.3 \mathrm{~g} \mathrm{~L}^{-1}$ bovine serum albumin (BSA) solution, the total flux decline rate (FDR) and the flux recovery ratio (FRR) of the membrane were $16.7 \%$ and $85.7 \%$ respectively, which indicated the good antifouling properties and application prospects of the TFC NF membrane.

\section{Introduction}

The requirement for clean water is expanding due to worsening water pollution and growing global population. Membrane technology has great potential for the application in water treatment and purification owing to its high efficiency as well as low energy consumption. Nanofiltration (NF), in which the molecular weight cut off (MWCO) for neutral organics is ranging from 150 to $2000 \mathrm{Da},{ }^{1}$ possesses unique advantages in separating divalent salts and low-molecular-weight organic from water compared with other pressure driven membranes such as ultrafiltration (UF) and reverse osmosis (RO). As a result, NF has broad application in desalination of seawater, reuse of sewage, food production, pharmaceutical and biotechnology industries. Interfacial polymerization (IP) is the most ordinary way to fabricate the thin-film composite (TFC) NF membranes while piperazine (PIP) and trimesoyl chloride (TMC) are the most widely used monomers in aqueous and organic phases, respectively. ${ }^{2}$ For the sake of better performance, many novel monomers are used to fabricate TFC membranes because the monomer has a decisive effect on membrane properties. Cheng et al. ${ }^{3}$ prepared a TFC membrane with amino-functional polyethylene glycol (PEG) and TMC by the IP process. The TFC membrane showed a pure water flux of

\footnotetext{
${ }^{a}$ State Key Laboratory of Chemical Engineering, Membrane Science and Engineering R\&D Lab, Chemical Engineering Research Center, East China University of Science and Technology, 130 Meilong Road, Shanghai 200237, China. E-mail: tangyongjian@ecust.edu.cn; Fax: +86-21-64252989; Tel: +86-21-64253670

${ }^{b}$ Jiangsu Zhenjiang Research Institute of Building Science Group CO., LTD, 8 Tanshan Road, Zhenjiang 212000, China
}

$66.0 \mathrm{~L} \mathrm{~m}^{-2} \mathrm{~h}^{-1}$ at 5 bar as well as good fouling resistance. Tang et al. ${ }^{4}$ fabricated TFC NF membranes with high chlorinetolerant properties using mixed aqueous monomers of PIP and 2,2'-bis(1-hydroxyl-1-trifluoromethyl-2,2,2-trifluoroethyl)4,4'-methylenedianiline (BHTTM). Furthermore, after preoxidation with $\mathrm{NaClO}$, the NF membrane achieved an ultrahigh permeability of $161 \mathrm{~L} \mathrm{~m}^{-2} \mathrm{~h}^{-1}$ while $\mathrm{Na}_{2} \mathrm{SO}_{4}$ rejection remained $92.1 \% .^{5}$

To satisfy the demand of industrial application, the poor fouling resistance of TFC NF membranes in long-time running must be resolved. The fouling occurs when foulants accumulate on the membrane surface and internal pores, leading to the permeability and rejection decline irreversibly. Antifouling property of membranes is closely related to surface roughness, electrostatic charges and hydrophilicity. ${ }^{6}$ Enhancing the hydrophilicity of membrane is a propitious method to heighten the antifouling property due to the hydrophobicity of many foulants and a thin water layer in the hydrophilic top layer is effective to keep the foulants from cumulating on the membrane. ${ }^{7}$ The highly hydrophilic TFC membranes can be fabricated by using hydrophilic monomers ${ }^{2,8,9}$ or blending inorganic nanoparticles. ${ }^{\mathbf{1 0}-14}$ Nevertheless, poor dispersity and aggregation of nanoparticles as well as the weak compatibility between polymer materials and inorganic nanoparticles limits the application of nanoparticles in fabricating TFC membrane. ${ }^{15}$ It is simple and effective to prepare highperformance TFC NF membranes by using hydrophilic monomers. Li et al. ${ }^{16}$ fabricated a sulfonated TFC membrane using 2,2'-benzidinedisulfonic acid (BDSA) and TMC which showed good application prospect in desalination of dye wastewater. The flux recovery ratio (FRR) of the membrane was $95.6 \%$ after 
washed with $20 \%$ alcohol solution, which indicated good antifouling property. $\mathrm{Lv}$ et al. ${ }^{17}$ synthesized a novel sulfonated aromatic diamine monomer, potassium 2,5-bis(4aminophenoxy)benzenesulfonate (BAPBS) to polymerize with TMC and the TFC membrane manifested good antifouling characteristics as well as high flux of $72.8 \mathrm{~L} \mathrm{~m}^{-2} \mathrm{~h}^{-1}$ at 6 bar. Weng et al. ${ }^{18}$ prepared three NF membranes using different aqueous monomers (PIP, $N$-aminoethyl piperazine (AEP) and zwitterionic $N$-aminoethyl piperazine propane sulfonate (AEPPS) respectively) and contrasted the membrane structure and performance, finding that the membrane prepared by AEPPS showed outstanding antifouling property and high flux of $80.3 \mathrm{~L} \mathrm{~m}^{-2} \mathrm{~h}^{-1}$ which was 3.8 and 8.1 times of the membranes made by AEP and PIP owing to its preeminent hydrophilicity and smooth surface structure.

In this work, using BDSA and PIP as aqueous monomers and TMC as organic monomer, TFC hollow fiber NF membrane was fabricated by IP. The structure and physicochemical characteristics of the NF membranes were analyzed by SEM, AFM, XPS and water contact angle. The separation properties of the NF membranes were analyzed by different salts and neutral organics. In addition, the antifouling performance was investigated through a long-term testing of bovine serum albumin (BSA).

\section{Experimental}

\subsection{Materials}

PES (polyethersulfone, $M_{\mathrm{w}}=58 \mathrm{kDa}$ ) was purchased from BASF company. $\mathrm{N}, \mathrm{N}$-Dimethylacetamide (DMAc, AR) was obtained from Xilong Scientific Co., Ltd. 2,2'-Benzidinedisulfonic acid (BDSA, $>70 \%$ ) was provided by TCI (Shanghai) Development Co., Ltd. Trimesoyl chloride (TMC, $\geq 98 \%$ ) was purchased from Qingdao Benzo Chemical Company (China). Inorganic salts including: $\mathrm{Na}_{2} \mathrm{SO}_{4}, \mathrm{MgSO}_{4}, \mathrm{MgCl}_{2}, \mathrm{NaCl}$, sodium hydroxide (NaOH, AR), n-butyl alcohol (AR), glucose (AR), sucrose (AR), raffinose (AR), bovine serum albumin (BSA, AR), diethylene glycol (DEG, AR), piperazine (PIP, GR) and $n$-hexane (AR) were purchased from Sinopharm Chemical Reagent Co. Ltd (China).

\subsection{Preparation of PES hollow fiber UF membranes}

The hollow fiber UF membranes were self-made in lab via using non-solvent induced phase inversion (NIPS) technique according to our previous report. ${ }^{19}$ The detailed spinning parameters were listed in Table 1 . The dope solution contained a certain

Table 1 Spinning conditions of PES hollow fiber membranes

Dope solution (wt\%)

Dope flow rate $\left(\mathrm{mL} \mathrm{min}^{-1}\right)$

Bore fluid composition

Bore fluid flow rate $\left(\mathrm{mL} \mathrm{min}^{-1}\right)$

External coagulant

Air gap $(\mathrm{cm})$

Nitrogen pressure (bar)

Dimension of spinneret $(\mathrm{mm})$

Spinneret temperature $\left({ }^{\circ} \mathrm{C}\right)$
PES/DMAc/DEG $(17: 43: 40)$

5

DI water

1.2

DI water

0

1.5

$\mathrm{ID} / \mathrm{OD}=1.4 / 0.7$

25 proportion of PES, DMAc and DEG was stirred by a magnetic stirrer for 12 hours and degassed overnight before spinning. The dope solution and bore fluid were pushed by nitrogen through the spinneret with the certain flow rates controlled by different pumps and the extruded membranes dropped into DI water by gravity for phase separation. The prepared hollow fiber membranes were immersed in DI water for 5 days to remove the residual solvents. The MWCO and PWP of the PES hollow fiber UF membranes were $70000 \mathrm{Da}$ and $150 \mathrm{~L} \mathrm{~m}^{-2} \mathrm{~h}^{-1} \mathrm{bar}^{-1}$, respectively.

\subsection{Preparation of TFC hollow fiber NF membranes}

A series of aqueous solutions containing different ratios of PIP/ BDSA (the total monomer concentration was $1 \% \mathrm{w} / \mathrm{v}$ and the concentration of $\mathrm{NaOH}$ was $0.05 \mathrm{~mol} \mathrm{~L}^{-1}$ ), and organic phase solutions with different concentration of TMC in $n$-hexane were prepared. The UF hollow fiber membranes were taken out of the DI water and both ends were sealed up to prevent the polymerization in the inwall of the hollow fiber membranes. The preparation process of TFC NF membrane was interpreted in Fig. 1. Firstly, the UF membrane was immersed in aqueous phase for 5 min after the water drops on the outside surface were blown away by an annular air knife. Then removed the droplets once again and immersed the membrane in organic phase for a certain time. After that, the membrane was immersed in $n$-hexane for $30 \mathrm{~s}$ to wash up the unreacted TMC. Finally, the NF membrane was hung up perpendicularly for 3 hours for the further polymerization.

\subsection{Membrane characterization}

XPS analysis. The chemical composition of the top surface on the PES UF membrane and TFC NF membranes was obtained by an X-ray photoelectron spectroscopy analysis (XPS, VG-miclab II, UK).

SEM analysis. The morphologies of the top surfaces and cross sections of the TFC membranes were analyzed by a scanning electron microscope (SEM, NOVA NANOSEM450) under $50000 \times$ magnification times. The samples were coated with gold before the SEM analysis.

AFM analysis. The surface morphologies and roughness of the TFC membranes were investigated by an atomic force microscopy (AFM, Veeco, NanoScope IIIa Multimode AFM). The scanning areas were $5 \mu \mathrm{m} \times 5 \mu \mathrm{m}$.

Dynamic water contact angle. The dynamic water contact angle of PES UF membrane and fabricated TFC NF membranes was measured by contact angle meter (JC2000A, Shanghai Zhong Cheng Digital Equipment Co., Ltd., China) at $25{ }^{\circ} \mathrm{C}$ to investigate the hydrophilicities.

\subsection{Nanofiltration performance of NF membranes}

Pure water flux was measured by using a lab-scaled stainless steel module. In each experiment, three modules were prepared under the same condition and tested in three identical crossflow set-ups with feed solutions circulated to make sure of the data reliability. The hydraulic pressure of feed solution was controlled through valves and the permeate solution was 


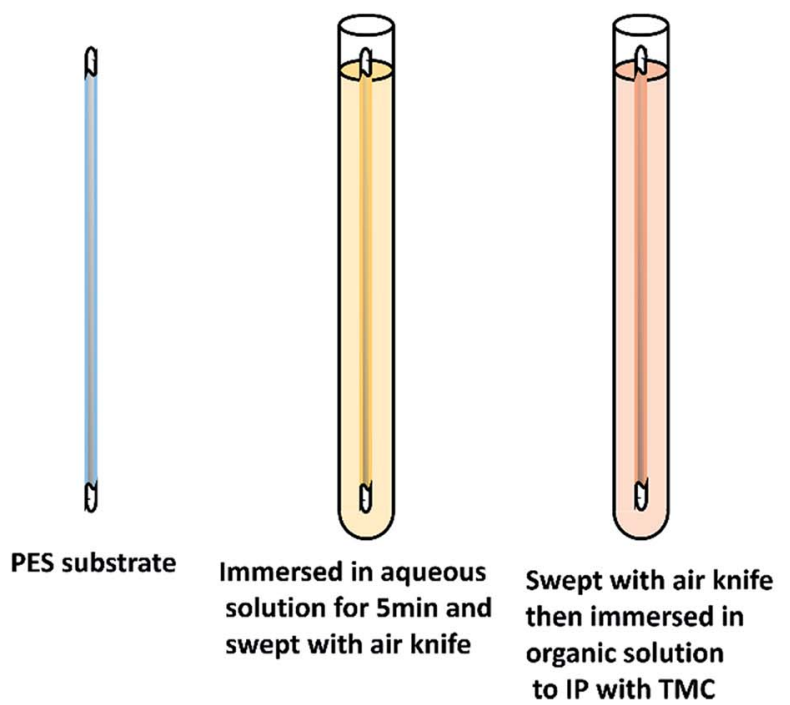

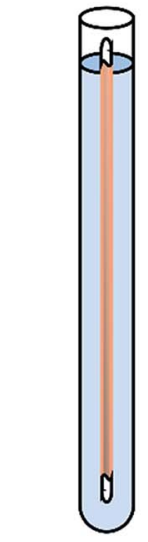

Immersed in $\mathbf{n}$-hexane

for $30 \mathrm{~s}$ to wash up the unreacted TMC

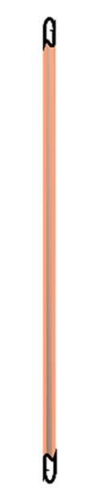

Hung up perpendicularly

for 3 hours

to IP with TMC

Fig. 1 Diagrammatic sketch of NF membrane preparation process.

collected. Permeate solutions were collected after deionized water circulating for $30 \mathrm{~min}$ under certain pressure. The computational formula of pure water permeability (PWP) was displayed as follow:

$$
\mathrm{PWP}=\frac{V}{A \times t}
$$

where $V$ was the volume of the permeate solution (L), $A$ was the effective membrane filtration area $\left(\mathrm{m}^{2}\right)$, and $t$ was the collecting time (h).

In the same way, the rejection performance was investigated with a series of salt solution $\left(\mathrm{Na}_{2} \mathrm{SO}_{4}, \mathrm{MgSO}_{4}, \mathrm{MgCl}_{2}\right.$ and $\mathrm{NaCl}$, $2000 \mathrm{ppm}$ ) and various neutral organic solutions ( $n$-butyl alcohol, glucose, sucrose and raffinose, $300 \mathrm{ppm}$ ).

The solute rejection was calculated according to following formula:

$$
R(\%)=\left(1-\frac{C_{\mathrm{p}}}{C_{\mathrm{f}}}\right) \times 100 \%
$$

where $C_{\mathrm{p}}$ and $C_{\mathrm{f}}$ represent the solute concentration (ppm) in permeate and feed solutions. The concentration of neutral organic solution was measured by a TOC analyzer (Shimadzu, Model TOCVPN, Japan) while salt concentration was measured by DDS-11A conductance meter (Shanghai Neici Instrument Company, China) and matched with the standard curve.

The MWCO of TFC NF membrane was evaluated by the rejection experiment of neutral organics and the molecular weight $\left(M_{\mathrm{w}}\right)$ of the solute which rejection was $90 \%$ was considered as the MWCO of the TFC membrane. The mean pore size and pore size distribution were evaluated with a log-normal model using MATLAB. The Stokes radius $\left(r_{\mathrm{s}}\right)$ was related with the probability of rejection and the geometrical mean radius of organic solute $\left(\mu_{\mathrm{s}}\right)$ was obtained at $R=50 \%$. The ratio of $r_{\mathrm{s}}$ at $R$ $=84.13 \%$ to that at $R=50 \%$ was defined as the geometrical standard deviation $\left(\sigma_{\mathrm{s}}\right)$. The mean pore radius $\left(\mu_{\mathrm{p}}\right)$ and pore size distribution $\left(\sigma_{\mathrm{p}}\right)$ were the same as $\mu_{\mathrm{s}}$ and $\sigma_{\mathrm{s}}$, respectively. The relationship between rejection of membrane and probability of rejection was calculated with the follow equation:

$$
\frac{\mathrm{d} R\left(r_{\mathrm{p}}\right)}{\mathrm{d} r_{\mathrm{p}}}=\frac{1}{r_{\mathrm{p}} \ln \sigma_{\mathrm{p}} \sqrt{2 \pi}} \exp \left[\frac{\left(\ln r_{\mathrm{s}}-\ln \mu_{\mathrm{s}}\right)^{2}}{2\left(\ln \sigma_{\mathrm{s}}\right)^{2}}\right]
$$

where $r_{\mathrm{p}}$ was the effective pore radius.

\subsection{Antifouling estimation of TFC NF membrane}

To investigate the antifouling property of TFC NF membranes, a long-term testing was carried out with a BSA solution $(0.3 \mathrm{~g}$ $\mathrm{L}^{-1}, \mathrm{pH} 7.0$ ) and the pressure was maintained at 4 bar. The experiment was implemented for three cycles. In each cycle, the DI water was filtrated through the membrane module for 4 hour, then the feed converted into BSA solution and operated for 8 hour and the permeability was recorded every hour. After three cycles test, the BSA solution was replaced with pure water, and the flux recorded over the following 4 hour.

Fouling caused by reversible resistances $\left(R_{\mathrm{r}}\right)$ and irreversible resistances $\left(R_{\mathrm{ir}}\right)$ was described by the following equation:

$$
\begin{gathered}
R_{\mathrm{r}}=\left(\frac{J_{\mathrm{w} 4}-J_{\mathrm{BSA} 3}}{J_{\mathrm{w} 0}}\right) \times 100 \% \\
R_{\mathrm{ir}}=\left(\frac{J_{\mathrm{w} 0}-J_{\mathrm{w} 4}}{J_{\mathrm{w} 0}}\right) \times 100 \%
\end{gathered}
$$

The total flux decline rate (FDR) and the flux recovery ratio (FRR) of the NF membrane after long-term testing was calculated according to the following equation:

$$
\begin{gathered}
\mathrm{FDR}=\left(1-\frac{J_{\mathrm{BSA} 3}}{J_{\mathrm{w} 0}}\right) \times 100 \% \\
\mathrm{FRR}=\frac{J_{\mathrm{w} 4}}{J_{\mathrm{w} 0}} \times 100 \%
\end{gathered}
$$


where $J_{\mathrm{w} 0}$ was the initial PWP, $J_{\mathrm{BSA} 3}$ was the permeability of BSA solution in the third cycle and $J_{\mathrm{w} 4}$ was the ultimate PWP.

\section{Results and discussions}

\subsection{Composition of the top surface of the NF membranes}

The chemical composition of the PES substrate and NF membranes was analyzed by XPS with range of 3-10 nm. Fig. 2 and Table 2 showed the XPS spectrum and the atom percent of the PES substrate and three TFC NF membranes, respectively. From the wide-scan spectra, nitrogen could be detected on the surface of TFC NF membranes, which indicating the polyamide layer was generated. There was a spot of sulfur on the top surface of the BDSA NF membrane which was similar to the result of $\mathrm{Hu}$ et $a .^{20}$ However, the peak of sulfur didn't exist in the spectrogram of the BDSA/PIP NF membrane. This may be explained that "- $\mathrm{SO}_{3} \mathrm{H}$ " was covered by the polymer chain as a result of interaction between BDSA and PIP. For the low molecular weight of PIP, it could permeate through the polyamide layer and continue to react with TMC, thus the " $-\mathrm{SO}_{3} \mathrm{H}$ " in the cross-linking chain was covered probably.
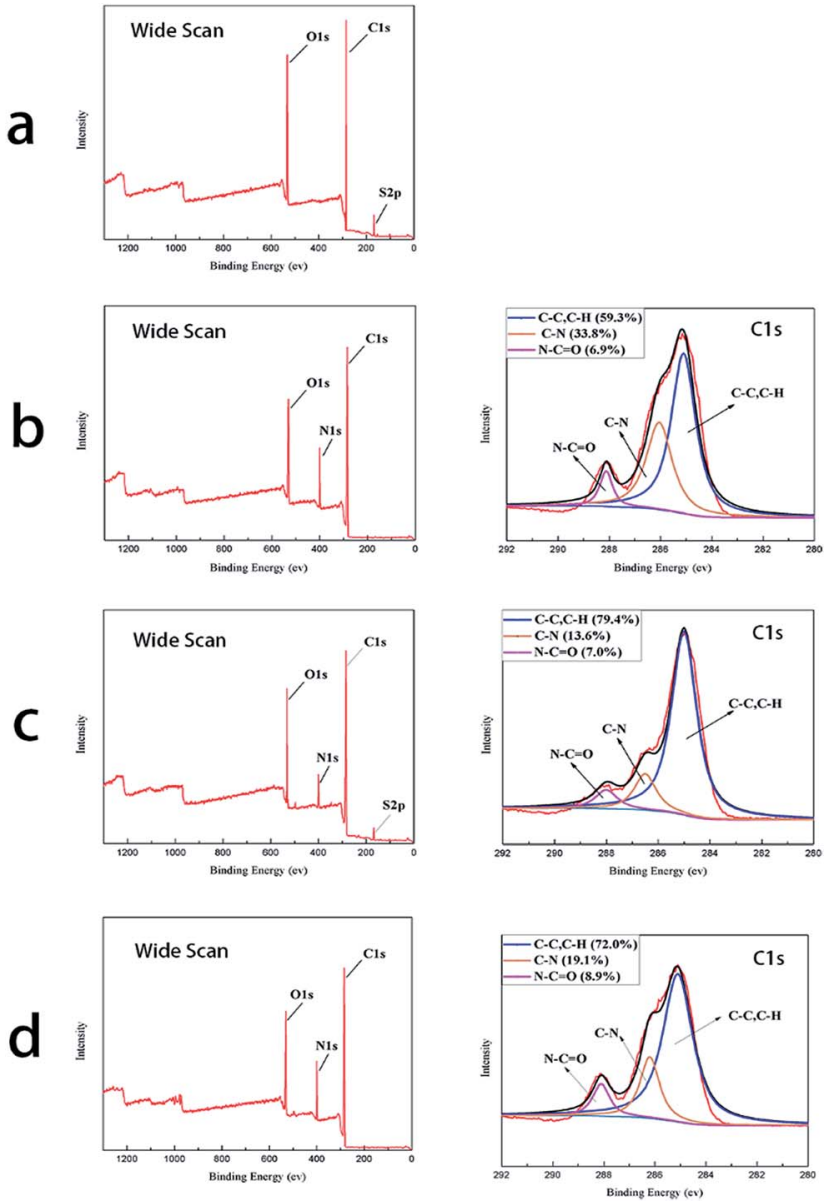

Fig. 2 XPS wide scan and C1 score-level of (a) the PES substrate, (b) the PIP NF membrane, (c) the BDSA NF membrane and (d) the BDSA/ PIP NF membrane.
Table 2 Elemental composition of the NF membranes

\begin{tabular}{llrll}
\hline & \multicolumn{4}{l}{ Atom percent $(\%)$} \\
\cline { 2 - 5 } Sample & C 1s & N 1s & O 1s & S 2p \\
\hline PIP & 73.83 & 11.46 & 14.71 & 0 \\
BDSA & 67.53 & 6.99 & 23.17 & 2.31 \\
BDSA/PIP & 73.04 & 11.91 & 15.05 & 0
\end{tabular}

The amount of carbon-carbon bond (C-C) and carbonhydrogen $(\mathrm{C}-\mathrm{H})$ was equal to the area of the peak at $285.1 \mathrm{eV}$. The peak at $286.1 \mathrm{eV}$ represented the carbon-nitrogen bond (C$\mathrm{N})$ which belonged to the unreacted amine in the aqueous phase. The content of amido bond $(\mathrm{N}-\mathrm{C}=\mathrm{O})$ illustrated the degree of polymerization. ${ }^{13}$ From the $\mathrm{C} 1$ score level spectra, the amido bond percent of the membrane prepared by mixed amine was larger than that of the PIP NF membrane and the BDSA NF membrane, indicating that the BDSA/PIP NF membrane hold a denser polyamide layer. The TFC membrane made from mixed amines hold a denser polyamide layer owing to the synergism effect between PIP and BDSA. ${ }^{21}$ For the mixing with BDSA, the polyamide backbone stiffness was enhanced. Therefore, the separation performance was improved.

\subsection{Morphology of the NF membranes}

Fig. 3 showed the morphologies of the top surfaces and cross sections of the PES substrate and TFC NF membranes. The image of PES substrate showed obvious sponge-like structure which was similar with the result in previous literatures and the
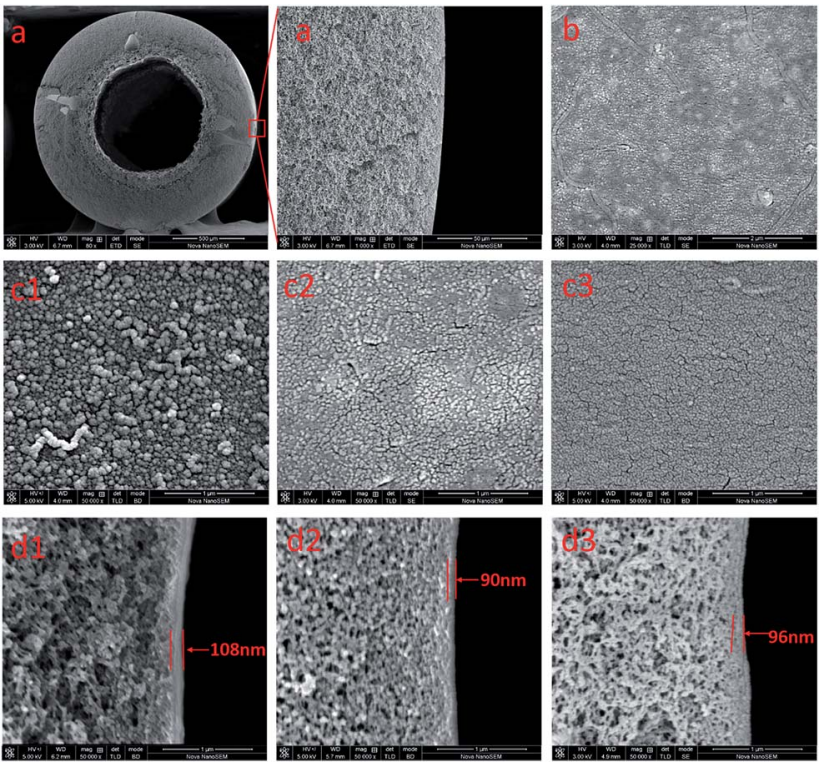

Fig. 3 SEM images of the top surfaces and cross sections of the substrate and NF membranes. (a) and (b) are the cross section and top surface of the PES substrate, respectively; (c1), (c2), (c3) and (d1), (d2), (d3) are the top surfaces and cross sections of the PIP NF membrane, the BDSA NF membrane and the BDSA/PIP NF membrane, respectively. 
spongy-like structure could provide appropriate pore size for IP progress and higher strength for high pressure operation. ${ }^{22,23}$ As shown in (c1), asymmetrical nodular structure could be observed in the surface of the PIP NF membrane which could be ascribed that the PIP with small molecular weight was easy to pass through the polyamide layer and react with TMC quickly forming aggregate in the top surface. ${ }^{24}$ The BDSA NF membrane had large ravines and gullies in the top surface while the BDSA/ PIP NF membrane was smoother than the PIP NF membrane and the BDSA NF membrane. From (d1)-(d3), it could be observed that all the TFC NF membranes had thin polyamide layers upon the PES substrate which were constructed by IP process. The difference of thickness between three NF membranes was limited. The BDSA/PIP NF membrane had a thickness of $96 \mathrm{~nm}$ which was between the PIP NF membrane (108 $\mathrm{nm}$ ) and the BDSA NF membrane (90 nm).

The AFM image of PES substrate and three NF membranes with an area of $5 \mu \mathrm{m} \times 5 \mu \mathrm{m}$ was shown in Fig. 4 . Compared with the PES substrate, all the TFC NF membranes had coarser surface. The BDSA/PIP NF membrane hold a smoothest surface which was tallied with the SEM result in Fig. 3. The PIP with smaller molecular weight was easier to move to the gap of polymer chain and react with acyl chloride group. Therefore the surface of the BDSA/PIP membrane was smoothest.

\subsection{Hydrophilicity of the NF membranes}

The hydrophilicity of the membranes has an important influence in membrane performance comprising antifouling property. Generally, the low contact angle (CA) emblematizes high hydrophilicity. The dynamic water contact angle $(\theta)$ of PES substrate and three TFC NF membranes was measured and shown in Fig. 5. The CA of the BDSA NF membrane was $67^{\circ}$ which was higher than that of PES substrate $\left(57^{\circ}\right)$. It could be explained that the BDSA molecule possessed some rigidity due to the biphenyl unit, thus the sulfonic acid groups were covered by the polymer skeleton. ${ }^{16}$ The hydrophilicity of PIP NF membranes promoted obviously compared with PES substrate because the IP process introduced amide groups as well as unreacted carboxyl and amino on the top surface. ${ }^{7,25}$ The CA of the BDSA/PIP NF membrane $\left(37^{\circ}\right)$ was higher than that of the PIP NF membrane $\left(28^{\circ}\right)$ because of the existence of hydrophobic
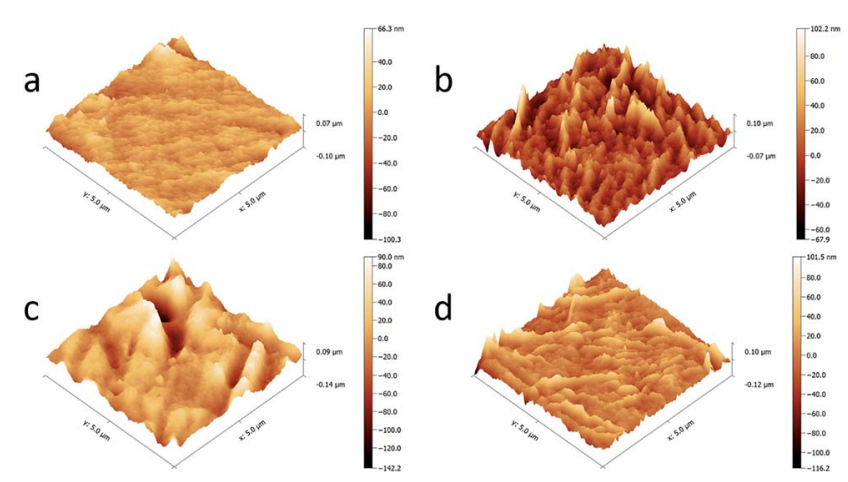

Fig. 4 AFM images of (a) the PES substrate, (b) the PIP NF membrane, (c) the BDSA NF membrane and (d) the BDSA/PIP NF membrane.

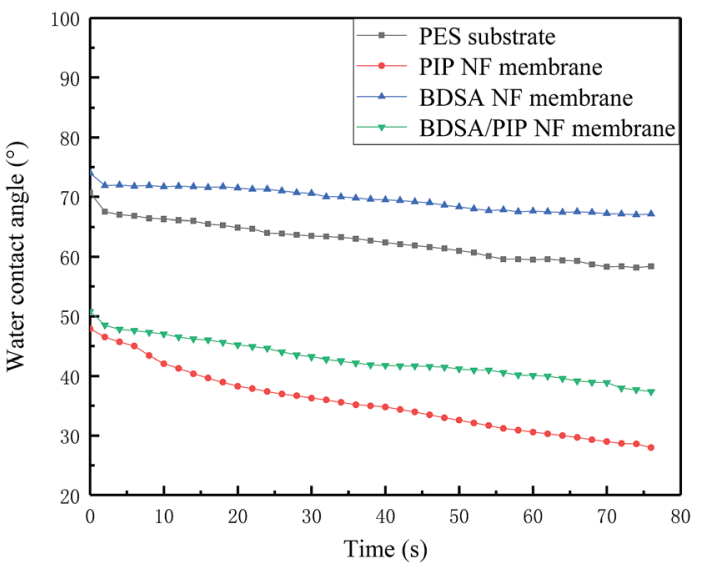

Fig. 5 Dynamic contact angle of PES substrate and NF membranes.

benzene rings in BDSA. Due to the interaction between PIP and BDSA, the hydrophilicity of the BDSA/PIP NF membrane improved remarkably compared with the BDSA NF membrane.

\subsection{Effect of preparation parameters on NF membrane performance}

TMC concentration. The influence of TMC concentration in organic phase on membrane separation performance was investigated. The aqueous monomer concentration $(1 \% \mathrm{w} / \mathrm{v}$, BDSA/PIP ratio was 6/4) and reaction time (30 s) remained unchanged. Fig. 6 showed the PWP and $\mathrm{Na}_{2} \mathrm{SO}_{4}$ rejection of $\mathrm{NF}$ membranes that prepared with different TMC concentration $(0.08,0.10,0.12$ and $0.14 \% \mathrm{w} / \mathrm{v})$. With the TMC concentration increased, the degree of polymerization enhanced and active layer became denser, ${ }^{26}$ the permeability decreased from $51.0 \mathrm{~L}$ $\mathrm{m}^{-2} \mathrm{~h}^{-1}$ to $49.1 \mathrm{~L} \mathrm{~m}^{-2} \mathrm{~h}^{-1}$ while the salt rejection increased from $84 \%$ to $95.5 \%$. With the TMC concentration kept on increasing, the permeability reduced distinctly but rejection raised slightly. Therefore, the optimal concentration of TMC was $0.12 \% \mathrm{w} / \mathrm{v}$.

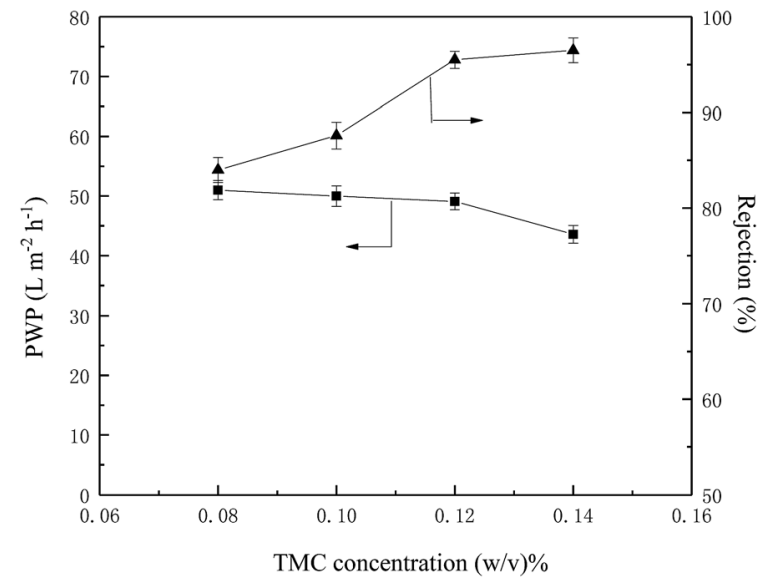

Fig. 6 Effect of TMC concentration on performance of NF membranes. 
Reaction time. The membrane performance depends on the structure and property of functional layer fabricated by IP process, thus the reaction time plays a significant role on preparing TFC NF membranes. With other preparation parameters remained unchanged, a series of NF membranes were prepared under different reaction time. The PWP and rejection to $\mathrm{Na}_{2} \mathrm{SO}_{4}$ were measured and illustrated in Fig. 7 . With the IP time increased from $10 \mathrm{~s}$ to $30 \mathrm{~s}$, the rejection increased from $68.2 \%$ to $95.5 \%$ while PWP decreased from $70.9 \mathrm{~L} \mathrm{~m}^{-2} \mathrm{~h}^{-1}$ to $49.1 \mathrm{~L} \mathrm{~m}^{-2} \mathrm{~h}^{-1}$. With reaction time expanded, the functional layer became denser so the rejection increased and permeability decreased obviously. When the reaction time exceeded $30 \mathrm{~s}$, the rejection decreased while permeability increased. This was attributed to the functional layer was dense enough thus prevented more monomers to achieve the reaction zone and led to the defective polymer structure. With further increase of reaction time, a great deal of acyl chloride groups on the membrane surface remained unreacted and hydrolyzed into carboxyl groups, thus the hydrophilicity of the membrane improved, causing the increment of permeability. ${ }^{26}$

BDSA/PIP ratio. There were two amine monomers in the aqueous phase and the ratio of BDSA/PIP was adjusted to fabricate NF membrane with prominent properties. Fig. 8 showed the permeability and $\mathrm{Na}_{2} \mathrm{SO}_{4}$ rejection of membranes fabricated under different BDSA/PIP ratio. The membrane prepared with BDSA showed a ultra-high flux but low rejection because the polymerization degree of the BDSA NF membrane was low. ${ }^{7}$ When PIP ratio in total amine $(1 \% \mathrm{w} / \mathrm{v})$ added from 0 to $40 \%$, the NF membrane expressed notable promotion in rejection while the permeability reduced. With the addition of PIP, the polymerization degree increased and the active layer became denser which could be corroborated by the XPS result in Fig. 2, which the " $\mathrm{N}-\mathrm{C}=\mathrm{O}$ " percent increased comparing with the BDSA NF membrane. By mixing the rigid BDSA with PIP, the backbone stiffness and microporosity of the polyamide layer increased which resulted in the increment of permeability. ${ }^{21}$ With PIP ratio increased from $40 \%$ to $50 \%$, the rejection reduced remarkably and permeability increased slightly. This could be ascribed to the competing effect between BDSA and PIP causing the decline of polymerization degree. With further

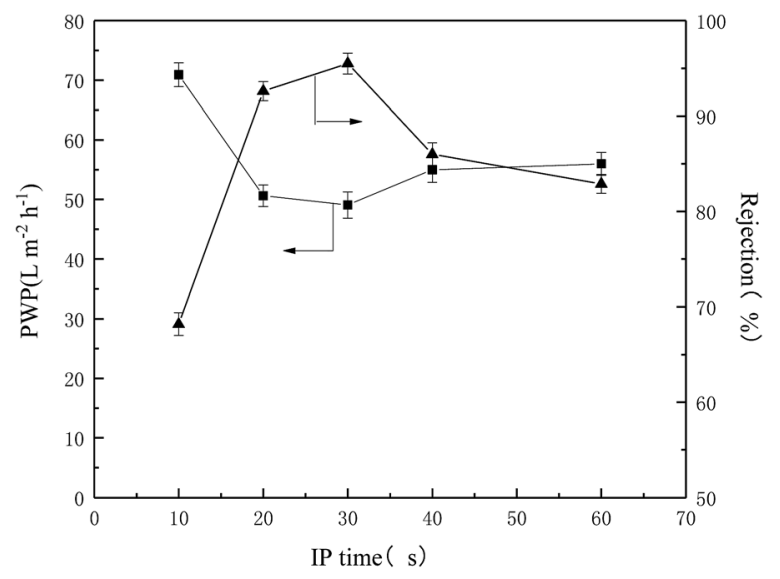

Fig. 7 Effect of reaction time on performance of NF membranes.

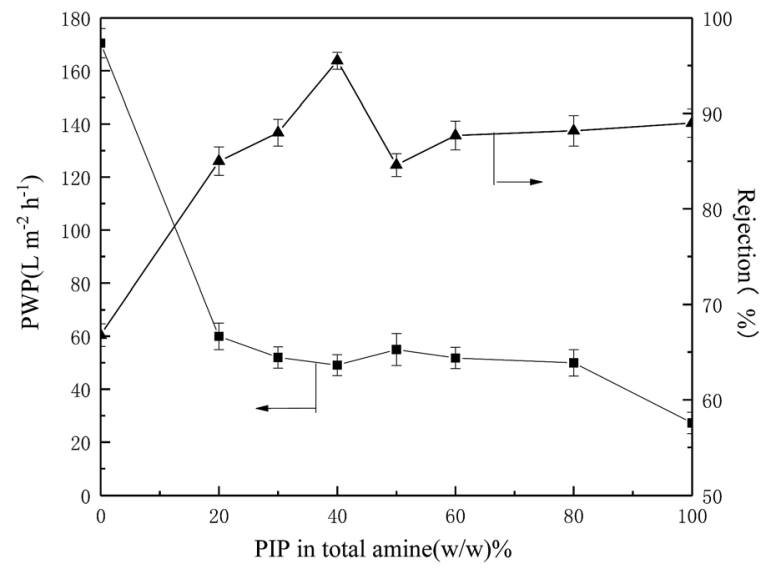

Fig. 8 Effect of PIP/BDSA ratio on performance of NF membranes.

increase of PIP/BDSA ratio, the rejection increased slowly while permeability decreased. The TFC membrane made in high PIP/ BDSA ratio showed the characteristic of the PIP NF membrane.

\subsection{Separation performance of the TFC NF membrane}

From the above discussion, the optimized preparation parameters could be obtained: $1 \% \mathrm{w} / \mathrm{v}$ amine (BDSA/PIP ratio 6/4) in aqueous solution, $0.12 \%$ TMC in organic solution and IP time $30 \mathrm{~s}$. The separation performance of three TFC membranes (the PIP NF membrane, the BDSA NF membrane and the BDSA/PIP NF membrane fabricated under the optimized conditions) was further investigated.

A series of neutral organic solutions ( $n$-butyl alcohol, glucose, sucrose and raffinose, $300 \mathrm{ppm}$ ) were used to test the BDSA/PIP NF membrane in order to confirm the pore size and MWCO, and the rejection of organics was displayed in Table 3. The MWCO and pore radius of the TFC membrane were $394 \mathrm{Da}$ and $0.52 \mathrm{~nm}$ respectively which were calculated by MATLAB. Fig. 9 was the pore size distribution curves.

The TFC NF membranes were tested with four inorganic salt solutions $\left(\mathrm{Na}_{2} \mathrm{SO}_{4}, \mathrm{MgSO}_{4}, \mathrm{MgCl}_{2}\right.$ and $\left.\mathrm{NaCl}, 2000 \mathrm{ppm}\right)$ to estimate the salt separating properties. The permeate flux and rejection of three TFC membranes to different salt solutions were exhibited in Fig. 10. The permeate flux of salt solutions was lower than PWP because of the concentration polarization. ${ }^{7}$ The permeate flux of the BDSA NF membrane was higher than the BDSA/PIP NF membrane and the PIP NF membrane. The sequence was consistent with the PWP in Fig. 8. The rejection of TFC membranes mainly followed the order: $\mathrm{Na}_{2} \mathrm{SO}_{4}>\mathrm{MgSO}_{4}>$

Table 3 Rejection to neutral organics of the BDSA/PIP NF membrane

\begin{tabular}{lllr}
\hline Solute & $\begin{array}{l}\text { Molecular weight } \\
(\mathrm{Da})\end{array}$ & $\begin{array}{l}\text { Stokes radius } \\
(\mathrm{nm})\end{array}$ & $\begin{array}{l}\text { Solute } \\
\text { rejection (\%) }\end{array}$ \\
\hline$N$-Butyl alcohol & 74 & 0.258 & $25 \pm 1.0$ \\
Glucose & 180 & 0.359 & $72.0 \pm 1.2$ \\
Sucrose & 342 & 0.462 & $88.2 \pm 1.1$ \\
Raffinose & 504 & 0.538 & $92.0 \pm 1.3$
\end{tabular}




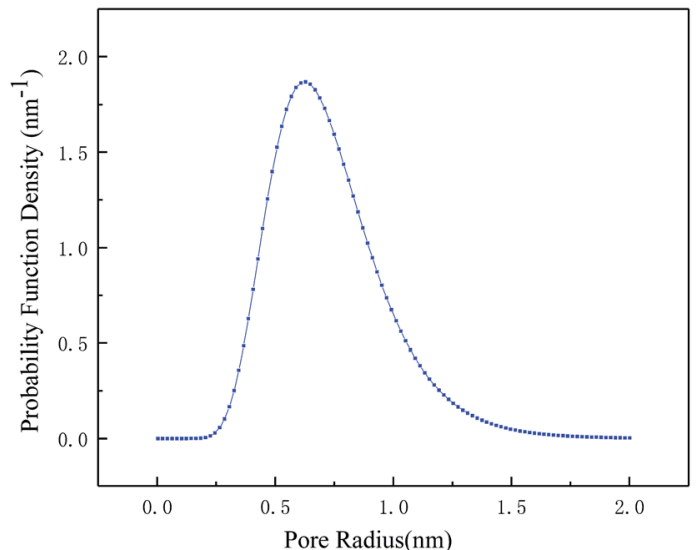

Fig. 9 Pore size distribution curves of the BDSA/PIP NF membrane.
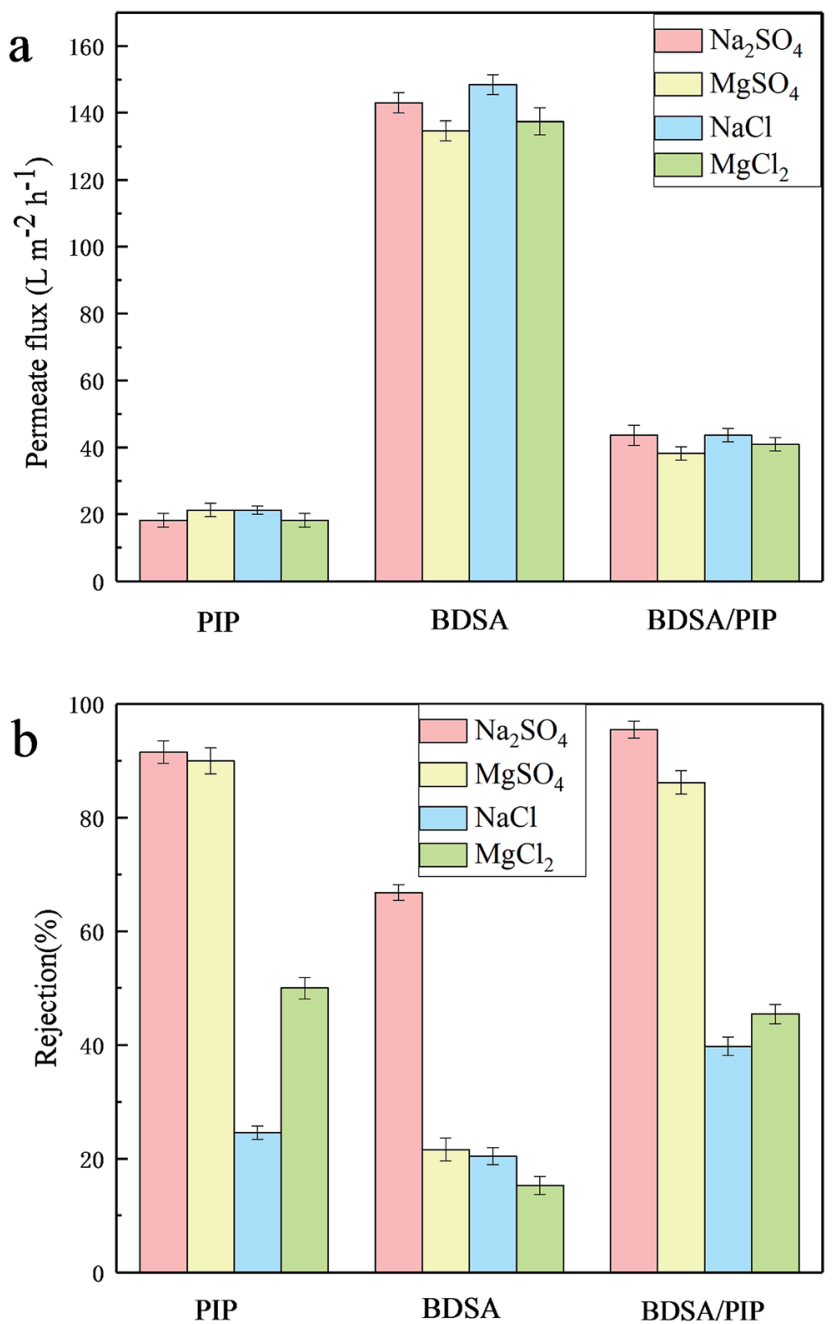

Fig. 10 Permeate flux (a) and rejection (b) of different inorganic salts for the TFC NF membranes.

$\mathrm{MgCl}_{2}>\mathrm{NaCl}$. The rejection to $\mathrm{Na}_{2} \mathrm{SO}_{4}$ and $\mathrm{MgSO}_{4}$ was higher than that of $\mathrm{NaCl}$ and $\mathrm{MgCl}_{2}$ because the anion with high valence was easier to be excluded by the negative surface than monovalent anion according to the Donnan exclusion. ${ }^{27}$ The rejection to $\mathrm{Na}_{2} \mathrm{SO}_{4}$ was higher than that of $\mathrm{MgSO}_{4}$. It was due to the combination between $\mathrm{Mg}^{2+}$ and the anions on the membrane surface, which would decrease the effective membrane surface charge, thus reducing the rejection performance. The rejection of the BDSA/PIP NF membrane and the PIP NF membrane was higher than the BDSA NF membrane owing to the higher cross-linking of the polyamide layer.

The permeation and rejection performance of TFC NF membrane under different pressures was shown in Fig. 11. It was obvious that the permeate flux increased linearly with pressure while the rejection to $\mathrm{Na}_{2} \mathrm{SO}_{4}$ fluctuated slightly. Therefore, the membrane showed the best performance under a pressure of 4 bar.

In addition, Table 4 listed the separating performance of various polyamide TFC membranes prepared with different monomers in aqueous phase in literatures and this work. By comparing these data, the BDSA/PIP NF membrane exhibited accredited flux as well as good rejection.

\subsection{Antifouling properties of the TFC NF membranes over long-term running}

A long-term testing consisted of three cycles was carried out to evaluate the antifouling performance of three TFC NF membranes. The permeability was measured every hour and the normalized flux $\left(J_{t} / J_{\mathrm{w} 0}\right)$ was illustrated in Fig. 12. The permeate flux declined notably when the feed solution changed to $0.3 \mathrm{~g}$ $\mathrm{L}^{-1}$ BSA in the first cycle and recovered to a certain degree after washed with DI water, while in the following testing, the TFC membrane showed a negligible decline in permeability. The $R_{\mathrm{r}}$, $R_{\text {ir }}$, FDR and FRR of the three membranes after three-cycles test were exhibited in Table 5. The BDSA/PIP NF membrane showed unfavourable performance in the fouling test while both the PIP NF membrane and the BDSA/PIP NF membrane expressed good antifouling performance. The antifouling performance of the TFC NF membranes was consistent with the water contact angle in Fig. 5. The hydrophilic membrane surface could build up a thin water layer to prevent the BSA deposition and adsorption. ${ }^{17}$ Furthermore, the pollutant was difficult to adsorb and

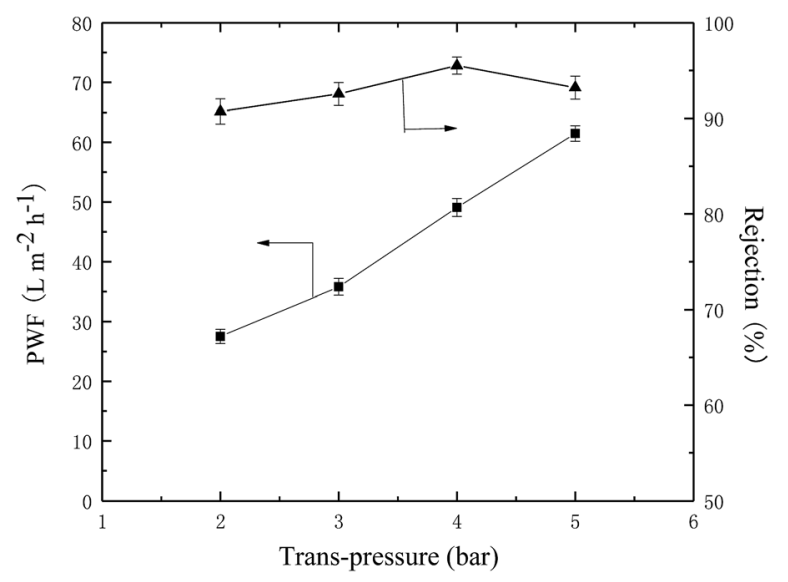

Fig. 11 The permeability and rejection of the BDSA/PIP NF membrane under various pressures. 
Table 4 Permeability and selectivity of various polyamide TFC membranes

\begin{tabular}{|c|c|c|c|c|c|c|c|c|}
\hline Monomers & $\begin{array}{l}\text { PWP } \\
\left(\mathrm{L} \mathrm{m}^{-2} \mathrm{~h}^{-1} \mathrm{bar}^{-1}\right)\end{array}$ & \multicolumn{4}{|c|}{ Salt rejection (\%) } & $\begin{array}{l}\text { Salt concentration } \\
(\mathrm{ppm})\end{array}$ & $\begin{array}{l}\text { Operation pressure } \\
\text { (bar) }\end{array}$ & Reference \\
\hline PEI & 6.4 & - & - & 98.0 & 84.0 & 1000 & 10 & 28 \\
\hline Amino-functional PEG & 13.2 & 45.8 & 80.2 & 95.4 & 54.8 & 2000 & 5 & 3 \\
\hline PEI + PIP & 5.1 & 50.0 & 74.0 & 92.0 & 65.0 & 500 & 8 & 29 \\
\hline
\end{tabular}

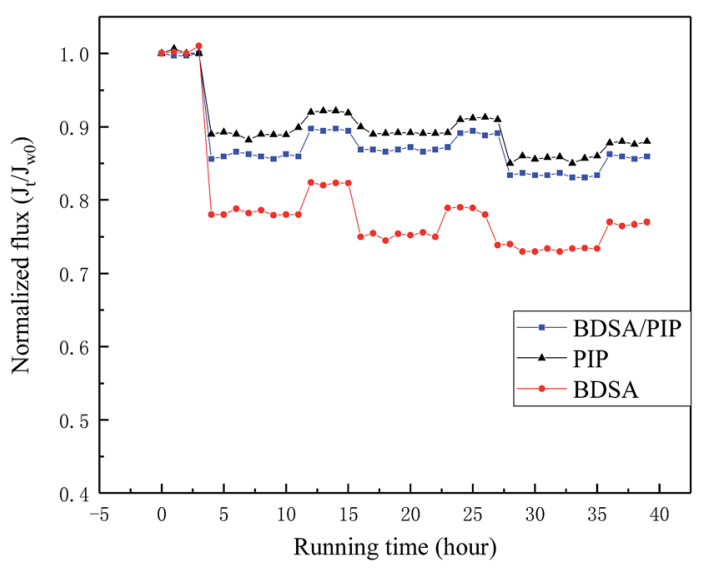

Fig. 12 Normalized flux of the TFC NF membranes in the antifouling test.

Table 5 Antifouling performance of the TFC NF membranes over long-term running

\begin{tabular}{lllll}
\hline Membrane & FRR $(\%)$ & FDR $(\%)$ & $R_{\mathrm{r}}(\%)$ & $R_{\text {ir }}(\%)$ \\
\hline PIP & 87.8 & 14.4 & 2.2 & 12.2 \\
BDSA & 76.8 & 26.7 & 3.5 & 23.2 \\
BDSA/PIP & 85.7 & 16.7 & 2.4 & 14.3 \\
\hline
\end{tabular}

accumulate in the smooth surface of the BDSA/PIP NF membrane. The result indicated good antifouling properties and application prospect of the BDSA/PIP NF membrane over long-term running.

\section{Conclusions}

A TFC hollow fiber NF membrane with high performance and good antifouling properties was fabricated with BDSA and PIP as mixed amine monomers in aqueous phase. The membrane preparation parameters including TMC concentration, reaction time and BDSA/PIP ratio were investigated and the optimized NF membrane showed a PWP of $49.1 \mathrm{~L} \mathrm{~m}^{-2} \mathrm{~h}^{-1}$ and $\mathrm{Na}_{2} \mathrm{SO}_{4}$ rejection of $95.5 \%$. The result of XPS, SEM and AFM showed that the BDSA/PIP NF membrane hold a denser and smoother surface than TFC membranes prepared with simplex monomer in aqueous phase. The rejection to different inorganic salts conformed to the following sequence: $\mathrm{Na}_{2} \mathrm{SO}_{4}>\mathrm{MgSO}_{4}>\mathrm{MgCl}_{2}$
$>\mathrm{NaCl}$. The MWCO and pore radius of the TFC membrane were about $400 \mathrm{Da}$ and $0.52 \mathrm{~nm}$ which were calculated by MATLAB simulation according to the rejection of neutral organics. A fouling test of $0.3 \mathrm{~g} \mathrm{~L}^{-1}$ BSA solution was carried out to investigate the antifouling properties of the TFC membrane, the FDR and FRR after long-term running were $16.7 \%$ and $85.7 \%$, respectively. The good separation performance and fouling resistance indicated comprehensive application potential of the TFC hollow fiber NF membrane.

\section{Conflicts of interest}

There are no conflicts to declare.

\section{Acknowledgements}

We acknowledge the financial support received from the National Science and Technology Support Project of China (2014BAB07B01 and 2015BAB09B01) and the Key Technology R\&D Program of Jiangsu Committee of Science and Technology in China (BE2013031).

\section{References}

1 P. Mou and S. D. Jons, Polymer, 2016, 103, 417-456.

2 Y. Pan, R. Xu, Z. Lü, S. Yu, M. Liu and C. Gao, J. Membr. Sci., 2017, 523, 282-290.

3 X. Q. Cheng, L. Shao and C. H. Lau, J. Membr. Sci., 2015, 476, 95-104.

4 Y. J. Tang, Z. L. Xu, S. M. Xue, Y. M. Wei and Y. Hu, J. Membr. Sci., 2016, 498, 374-384.

5 Y.-J. Tang, L.-J. Wang, Z.-L. Xu, Y.-M. Wei and H. Yang, J. Membr. Sci., 2016, 502, 106-115.

6 D. Rana and T. Matsuura, Chem. Rev., 2010, 110, 2448-2471.

7 A. Akbari, E. Aliyarizadeh, S. M. Mojallali Rostami and M. Homayoonfal, Desalination, 2016, 377, 11-22.

8 W. Li, C. Bian, C. Fu, A. Zhou, C. Shi and J. Zhang, J. Membr. Sci., 2016, 504, 185-195.

9 Q. An, F. Li, Y. Ji and H. Chen, J. Membr. Sci., 2011, 367, 158165.

10 Y. T. Chung, E. Mahmoudi, A. W. Mohammad, A. Benamor, D. Johnson and N. Hilal, Desalination, 2017, 402, 123-132.

11 J. Zheng, M. Li, K. Yu, J. Hu, X. Zhang and L. Wang, J. Membr. Sci., 2017, 524, 344-353. 
12 M. Yang, C. Zhao, S. Zhang, P. Li and D. Hou, Appl. Surf. Sci., 2017, 394, 149-159.

13 S. M. Xue, Z. L. Xu, Y. J. Tang and C. H. Ji, ACS Appl. Mater. Interfaces, 2016, 8, 19135-19144.

14 Z. Geng, X. Yang, C. Boo, S. Zhu, Y. Lu, W. Fan, M. Huo, M. Elimelech and X. Yang, J. Membr. Sci., 2017, 529, 1-10.

15 J. Zhu, L. Qin, A. Uliana, J. Hou, J. Wang, Y. Zhang, X. Li, S. Yuan, J. Li, M. Tian, J. Lin and B. Van der Bruggen, ACS Appl. Mater. Interfaces, 2017, 9, 1975-1986.

16 M. Li, Y. Yao, W. Zhang, J. Zheng, X. Zhang and L. Wang, Environ. Sci. Technol., 2017, 51, 9253-9260.

17 Z. Lv, J. Hu, J. Zheng, X. Zhang and L. Wang, Ind. Eng. Chem. Res., 2016, 55, 4726-4733.

18 X. Weng, Y. Ji, F. Zhao, Q. An and C. Gao, RSC Adv., 2015, 5, 98730-98739.

19 B.-W. Zhou, H.-Z. Zhang, Z.-L. Xu and Y.-J. Tang, Desalination, 2016, 394, 176-184.

$20 \mathrm{~J} . \mathrm{Hu}, \mathrm{Z} . \mathrm{Lv}$, Y. Xu, X. Zhang and L. Wang, J. Membr. Sci., 2016, 505, 119-129.
21 Y.-J. Tang, Z.-L. Xu, S.-M. Xue, Y.-M. Wei and H. Yang, J. Membr. Sci., 2017, 541, 483-491.

22 J. Gao, S.-P. Sun, W.-P. Zhu and T.-S. Chung, J. Membr. Sci., 2016, 499, 361-369.

23 H.-Z. Zhang, Z.-L. Xu, Y.-J. Tang and H. Ding, J. Membr. Sci., 2017, 527, 111-120.

24 Y.-J. Tang, Z.-L. Xu, B.-Q. Huang, Y.-M. Wei and H. Yang, RSC Adv., 2016, 6, 45585-45594.

25 H.-Z. Zhang, Z.-L. Xu, H. Ding and Y.-J. Tang, Desalination, 2017, 420, 158-166.

26 X. Li, Y. Cao, H. Yu, G. Kang, X. Jie, Z. Liu and Q. Yuan, J. Membr. Sci., 2014, 466, 82-91.

27 H. Tang, J. He, L. Hao, F. Wang, H. Zhang and Y. Guo, J. Membr. Sci., 2017, 524, 612-622.

28 Z. Thong, Y. Cui, Y. K. Ong and T.-S. Chung, ACS Sustainable Chem. Eng., 2016, 4, 5570-5577.

29 D. Wu, S. Yu, D. Lawless and X. Feng, React. Funct. Polym., 2015, 86, 168-183. 